\title{
Selected Abstracts From the Wilderness Medical Society's 2009 Annual Scientific Meeting
}

\section{Avalanche Beacon Reception Under Water: A Pilot Study Applying Avalanche Beacon Technology to Improve Scuba Diver Situational Awareness}

Lost, disoriented, or entangled scuba divers are at risk of asphyxiation due to low air supply: time-sensitive situations that depend on timely "buddy rescue." Similarly, avalanche victims die most frequently from asphyxiation if not rescued by a nearby buddy, with survival probability decreasing quickly, from $91 \%$ at 18 minutes after burial to $34 \%$ at 35 minutes. In these situations where elapsed time until rescue is one of the most critical variables of survival, using an avalanche beacon has resulted in a lower median duration of burial ( 5 minutes vs 125 minutes; $P<.001)$ and rate of mortality $(55.2 \%$ vs $70.6 \%$; $P<.001$, odds ratio 0.26 ) when compared to avalanche victims not using a beacon. This knowledge and technology have made avalanche beacons part of standard backcountry travel gear and are now helping firefighters locate lost comrades and build exits when visual communication is impaired.

Scuba divers in low visibility situations may also benefit from a personal, hand-held device that helps locate their buddy if visual communication is lost. Modern day avalanche beacons may serve this purpose.

Initial testing was carried out in the shallow end (3-foot depth) of a local 100 -foot by 50 -foot swimming pool. Four submerged, identical, opaque, watertight lexan cases were submerged to the bottom in the four corners of the pool's shallow end. One of the cases contained a Tracker DTS avalanche beacon in "transmit" mode. Volunteers used an identical avalanche beacon in "search" mode, and encased in a similar but clear, water-tight case, to identify which of the 4 cases contained the missing beacon. Ten out of 10 volunteers, each with minimal training and within 1 minute 30 seconds, were able to use the "tracking" beacon to locate the "missing" beacon. Avalanche beacon reception under water is possible and warrants further study applying its technology to underwater applications.

Jon S. Solberg, MD

David C. Hile, MD

Ft. Lewis, WA, USA

\section{The Ultrasound Identification of Simulated Long Bone} Fractures by Prehospital Providers

Objective.-In the wilderness and other austere environments, decisions regarding patient management are often limited by obtainable resources. Portable ultrasound may allow for the detection of fractures in areas where other imaging modalities such as radiography are not readily available. We used a simulation training model for the ultrasound diagnosis of long bone fractures to study the ability of emergency medical technicians (EMTs) to detect the presence or absence of a variety of simulated fracture patterns using a portable ultrasound.

Methods.-The fracture simulation model is composed of a bare turkey leg bone that is mechanically fractured and housed in a shallow plastic container within a completely opaque gelatin base solution. Five different fracture patterns were created: no fracture, transverse fracture, segmental fracture, oblique fracture, and comminuted fracture. Twenty EMTs then sonographically evaluated these 5 models with a SonoSite M-Turbo portable ultrasound device for the presence or absence of a fracture.

Results.-EMTs correctly identified the presence or absence of a fracture in the models with no fracture, a transverse fracture, and an oblique fracture $95 \%$ of the time. Participants always correctly identified the presence of a fractured model when assessing the comminuted and segmental fracture model. Across detection of all fracture patterns, a final sensitivity of 97.5\% (95\% confidence interval [CI]: 94.1\%-100.0\%) and a specificity of $95.0 \%$ (95\% CI: $85.4 \%-100.0 \%$ ) was observed in our study.

Conclusions.-Using a portable ultrasound device, EMTs were able to correctly detect the presence or absence of a simulated long bone fracture with a high degree of sensitivity and specificity. Future studies may further evaluate the ability of other groups to use ultrasound to assist in the diagnosis of fractures, as well as examine the clinical impact of this skill in environments where conventional imaging modalities are limited or unavailable.

Jason D. Heiner, MD

Michael D. Perreault, MD

Todd J. McArthur, MD

Tacoma, WA, USA

\section{Predictive Factors of Successful Summiting on Aconcagua}

Background.-Base camp physicians and expedition leaders commonly measure static vital signs when screening mountaineers prior to summit attempts. Single measurements of functional status such as the 6-minute walk test (6MWT) and postexercise vital signs (VS) have not been described at high altitude.

Objective.-Determine if postexercise VS or 6-minute walk distance (6MWD) predicts successful summiting at extreme altitude on Aconcagua.

Methods.-Prospective observational cohort study. Setting: Plaza de Mulas base camp (4365 m) Aconcagua Provincial Park, Argentina. Inclusion criteria: volunteer trekkers to 